\title{
Atividade ovicida e larvicida do extrato etanólico de Aloe vera L. sobre Haemonchus contortus*
}

\author{
Ovicidal and larvicidal activity of Aloe vera L. ethanolic extract against \\ Haemonchus contortus
}

\author{
Lew Kan Sprenger**
}

\begin{abstract}
Resumo
O objetivo deste trabalho foi determinar a eficácia in vitro do extrato etanólico de Aloe vera L. frente a estágios de vida livre de Haemonchus contortus. O fitoterápico foi produzido com percolação da polpa do vegetal com álcool absoluto, a $25^{\circ} \mathrm{C}$, sendo posteriormente liofilizado. Foram realizados o teste de eclodibilidade de ovos (TEO) e o teste de desenvolvimento larval (TDL), com concentrações crescentes $(0,78$ a $100 \mathrm{mg} / \mathrm{mL})$ em seis repetições. Para analisar a composição química do fitoterápico, procedeuse a marcha fitoquímica completa, analisando qualitativamente a presença de fenóis, taninos, antocianinas, antocianidinas, leucoantocianidinas, estero ides, triterpenos, saponinas, resinas e alcaloides; além da quantificação de fenóis totais, teste de toxicidade frente Artemia salina e ensaio antioxidante pelo teste de redução do radical DPPH. O produto obtido a partir de $A$. vera apresentou eficácia de $94,35 \pm 1,13 \%$ no TEO, já no TDL a eficácia foi de 76,03 \pm 0,45\%, ambos na concentração de $100 \mathrm{mg} / \mathrm{mL}$. Nas análises fitoquímicas, foram encontrados diversos compostos que podem ter contribuído com o efeito anti-helmíntico, tanto direta como indiretamente. Os dados da marcha fitoquímica, aliados aos resultados dose-dependentes obtidos nos testes in vitro evidenciam que o extrato produzido possui potencial para combater $H$. contortus. Novos estudos devem ser realizados buscando maximizar a eficácia deste extrato, uma vez que foram encontrados resultados excelentes nos testes realizados.
\end{abstract}

Palavras-chave: Etnoveterinária, fitoterapia, infecção parasitária.

\begin{abstract}
The aim of this study was to determine the in vitro efficacy of ethanolic extract of Aloe vera L. against Haemonchus contortus free-living stages. The herbal material was produced by pulp leaving the solution with ethanol at $25^{\circ} \mathrm{C}$ and latter the material was lyophilized. After this, the product was used for the egg hatch test (EHT) and the larval development test (LDT) in different concentrations ( 0.78 to $100 \mathrm{mg} / \mathrm{mL}$ ) with six replicates. A complete phytochemical screening was performed to analyze the materials' chemistry composition, screening qualitatively the presence of phenols, tannins, anthocyanins, anthocyanidins, leucoanthocyanidins, steroids, triterpenes, saponins, resins and alkaloids; beyond the total phenols quantification, brine shrimp toxicity test and DPPH radical reduction antioxidant test. $A$. vera extract had an efficacy of $94,35 \pm 1,13 \%$ using the EHT and of $76,03 \pm 0,45 \%$ when using the LDT test, both in concentration $100 \mathrm{mg} / \mathrm{mL}$. Phytochemical tests showed few chemical compounds that could exert anthelmintic properties. The results obtained with the biochemical tests together with the dose-dependent effect found in in vitro tests demonstrate that extract has the potential to be a good drug candidate against $H$. contortus. Further studies should be conducted to maximize the effectiveness of the herbal, because it demonstrated excellent results in experiments.
\end{abstract}

Keywords: Ethnoveterinary, herbal medicine, parasitic infection.

\section{Introdução}

A produção de pequenos ruminantes no Brasil cresce anualmente, contribuindo de forma expressiva para o balanço mercantil do país (Sprenger et al., 2013). Apesar do enorme potencial, alguns entraves sanitários diminuem a produtividade, sendo o principal deles as parasitoses gastrintestinais, que geram déficits econômicos significativos (Molento et al., 2013). A infecção por Haemonchus contortus é a principal doença parasitária, causando prejuízos devido a anorexia, apatia, aumento da conversão alimentar, diminuição da produção e perda de peso em animais infectados (Nery et al., 2010).

O controle da enfermidade é, geralmente, realizado de forma intensiva, com o uso indiscriminado de anti-helmínticos comerciais, sem considerar os fatores epidemiológicos envolvidos. Este modelo de utilização favorece a seleção e a propagação da população parasitária resistente, além da deposição de resíduos no meio ambiente e aumento dos custos produtivos (Molento, 2005).

Assim sendo, diversos estudos buscando novas terapias alternativas, sendo o uso de produtos fitoterápicos o que se encontra em fase mais avançada de pesquisa e demonstrando resultados promissores (Sprenger et al., 2015). Dentre as diversas plantas que estão sendo estudas atualmente, Aloe vera L., popularmente conhecida como babosa, destaca-se devido às suas propriedades medicinais (Huseini et al., 2012). O extrato produzido a partir da planta apresenta efeito comprovado no tratamento da osteoartrite, diabetes (Rajasekaran et al., 2006);

\footnotetext{
*Recebido em 25 de junho de 2015 e aceito em 25 de outubro de 2015.

**Universidade do Contestado (UnC), Campus Canoinhas, Departamento de Medicina Veterinária. R. Roberto Ehlke 86, Centro, Canoinhas, SC, 89460-000,

Brasil. Correspondência: lew.sprenger@gmail.com
} 
úlceras estomacais, febre e inflamações (Ramos e Pimentel, 2013); infecções bacterianas (Cellini et al., 2014), infecções por Eimeria sp. (Yim et al., 2011).

O presente estudo foi desenvolvido com o objetivo de avaliar a eficácia do extrato hidroalcoólico de $A$. vera em fases de vida livre de $H$. contortus, utilizando testes in vitro.

\section{Material e métodos}

O estudo foi realizado no laboratório de microbiologia da Universidade do Contestado, Campus Canoinhas, durante o mês de maio, 2015. Amostras de folhas de $A$. vera $\mathrm{L}$. foram obtidas no Mercado Municipal de Curitiba, Paraná, autentificada pela confirmação por botânicos da Universidade Federal do Paraná (UFPR) e depositada uma amostra representativa no herbário da mesma instituição. Imediatamente após a obtenção, as folhas foram lavadas com água clorada (10ppm de cloro), seguido por água destilada. Depois disso, a polpa foi recolhida com auxílio de uma espátula e foi processada para a produção dos extratos aquoso e etanólico.

Para a produção do extrato etanólico, $100 \mathrm{~g}$ da polpa foram misturadas com $1 \mathrm{~L}$ de etanol absoluto (95\%; Merck®, Alemanha), durante $10 \mathrm{~min}$ a $4^{\circ} \mathrm{C}$. A suspensão foi homogeneizada e agitada vigorosamente em agitador magnético à temperatura ambiente $\left(25^{\circ} \mathrm{C}\right)$. Transcorrido este tempo, o material foi filtrado, com auxílio de uma bomba de vácuo, em funil com papel filtro. $\mathrm{O}$ extrato foi concentrado em evaporador rotatório, sob pressão reduzida e à temperatura de $28^{\circ} \mathrm{C}( \pm 2)$, para posteriormente liofilização. O rendimento do extrato etanólico seco foi de $21 \mathrm{~g}$.

Posteriormente se realizou a marcha fitoquímica qualitativa descrita por Matos (1998) com o objetivo de verificar a presença de metabólitos secundários (fenóis, taninos, antocianinas, antocianidinas, leucoantocianidinas, esteroides, triterpenos, saponinas, resinas e alcaloides). A dosagem de fenóis totais (FT) foi realizada pelo teste de Folin-Ciocalteu, seguindo a metodologia adaptada por McDonald et al. (2001). O teor de FT foi determinado por interpolação da absorbância das amostras contra a curva de calibração construída com padrões de ácido gálico. Os resultados, determinados a partir da equação de regressão da curva de calibração ( $\left.y=0,02 x-0,0063 ; R^{2}=0,9442\right)$, foram expressos em miligramas de ácido gálico equivalentes por grama da amostra. O ensaio antioxidante foi realizado pelo método de redução do radial livre DPPH (1,1-difenil-2picril-hidrazila), de acordo com a metodologia de Blois (1958), usando como padrão o ácido ascórbico. A atividade toxicidade do extrato foi avaliada através do teste de letalidade frente Artemia salina Leach, seguindo a metodologia de Meyer et al. (1982). Como critério de toxicidade dos extratos foi estabelecido que

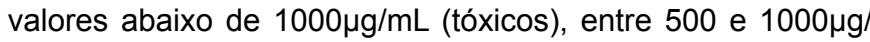
$\mathrm{mL}$ (fracamente tóxico) e acima de $1000 \mu \mathrm{g} / \mathrm{mL}$ (não tóxicos).

Para a realização do teste de eclodibilidade larval (TEO), inicialmente se procedeu à recuperação dos ovos. Para tal, foram colhidas fezes diretamente da ampola retal de caprinos, previamente selecionados, com contagem de OPG superior a 2800 . Um pool de $55 \mathrm{~g}$ de fezes foi processado conforme o protocolo de Coles et al. (1992), adaptado por Bizimenyera et al. (2006). Para seguir a adaptação, aproximadamente $55 \mu \mathrm{L}$ da suspensão contendo 200 ovos foram acondicionados em cada poço em placas de 24 poços. Calculado o volume final de $1 \mathrm{~mL}$ para cada poço, os tratamentos fitoterápicos foram preparados nas concentrações de: $100 ; 50 ; 25 ; 12,5 ; 6,25 ; 3,125 ; 1,562$ e $0,781 \mathrm{mg} / \mathrm{mL}$. O extrato foi diluído com $3 \%$ de DMSO. O controle negativo foi feito com água destilada; já os controles positivos foram realizados com o emulsificante DMSO aquoso a $3 \%(\mathrm{v} / \mathrm{v}) \mathrm{e}$ o outro com albendazol $0,63 \mathrm{mg} / \mathrm{mL}$. A contagem dos ovos e das larvas foi realizada em microscópio invertido. Seis réplicas foram realizadas para cada tratamento e também para os controles. No cálculo da eficácia, utilizou-se a seguinte fórmula:

Percentual de eclodibilidade $(\%)=\mathrm{L} 1 /$ (ovos $+\mathrm{L} 1) \times 100$

No teste de desenvolvimento larval (TDL), para a obtenção das larvas de primeiro estádio (L1), obteve-se uma alíquota de suspensão de ovos, conforme método descrito por Hubert e Kerboeuf (1992), a qual posteriormente foi incubada por 24 horas em estufa a $37^{\circ} \mathrm{C}$. O TDL foi realizado seguindo-se a metodologia descrita por Roberts e O'Sullivan (1950), modificado. Uma alíquota de $1 \mathrm{~mL}$, contendo aproximadamente $230 \mathrm{~L} 1 \mathrm{de} H$. contortus, foi incubada por 6 dias com $2 \mathrm{~g}$ de fezes de um animal livre de infecção parasitária, juntamente com $1 \mathrm{~mL}$ do extrato nas concentrações $100 ; 50 ; 25 ; 12,5 ; 6,25 ; 3,125 ; 1,562$ e $0,781 \mathrm{mg} / \mathrm{mL}$. O controle negativo foi feito com água destilada, já os controles positivos foram realizados com o emulsificante DMSO aquoso a $3 \%(\mathrm{v} / \mathrm{v})$ e o outro com ivermectina $(0,63 \mathrm{mg} /$ $\mathrm{mL})$. Seis réplicas foram realizadas para cada tratamento e também para os controles.

A análise estatística foi realizada com a análise de variância (ANOVA), seguido do teste de Tukey, onde foram considerados estatisticamente diferentes os resultados que apresentaram probabilidade de ocorrência da hipótese de nulidade menor que $5 \%(p<0,05)$. Todas as análises foram realizadas usando-se o programa GraphPad Prism ${ }^{\circledR} 5$.

\section{Resultados e discussão}

Na busca por fitoterápicos antiparasitários, os testes in vitro são fundamentais para fazer uma análise preliminar dos compostos químicos e sua ação. Tendo em vista a grande quantidade de herbais sendo pesquisados, os métodos in vitro destacam-se por seu baixo custo e tempo empregados, além de não serem utilizados animais vertebrados experimentais (Fortes et al., 2014).

$\mathrm{Na}$ marcha fitoquímica, foram detectadas a presença de alcaloides, catequinas, esteroides, fenóis, resinas e taninos. Antocianinas, antocianidinas, leucoantocianidinas e triterpenos não foram encontradas nos testes usados. O teor de fenóis totais, em equivalentes de ácido gálico, foi de $0.184 \pm 0.021 \mathrm{mg} / \mathrm{g}$. $\mathrm{O}$ teste de toxicidade frente $A$. salina apresentou DL50 de $1.785 \mu \mathrm{g} / \mathrm{mL}\left(R^{2}=0,8875\right)$. No ensaio antioxidante foi observado que a $\mathrm{CE}_{50}$, concentração na qual ocorre $50 \%$ do efeito, foi 47,55 $\pm 0,99 \mathrm{mg} / \mathrm{mL}$, já a do padrão foi $0,5 \pm 0,03 \mathrm{mg} / \mathrm{mL}$.

Na marcha fitoquímica, os compostos encontrados nos testes qualitativos corroboram com os expostos na literatura científica (Dagne et al., 2000). Dentre os principais responsáveis pela atividade anti-helmíntica de $A$. vera, destacam-se os flavonoides e taninos (Kayser et al., 2003). Além destes, a planta possui outros metabólitos capazes de produzir respostas pró-inflamatórias, as quais são importantes para combater os desafios sanitários enfrentados pelos animais (Cellini et al., 2014). 
Os taninos possuem efeito anti-helmíntico pronunciado, devido principalmente à diminuição da disponibilidade de nutrientes ao organismo do parasita (Hoste et al., 2006). Especificamente para helmintos, também ocorre redução da fertilidade das fêmeas adultas e da eclodibilidade dos seus ovos. Além disso, há diminuição da motilidade das larvas, o que pode diminuir a taxa de contaminação da pastagem (Molan et al., 2003). Como efeito secundário, acontece uma maior biodisponibilidade de proteínas no organismo do animal, podendo levar a uma maior resposta imune sobre parasitos intestinais (Alonso-Díaz et al., 2011). Athanasiadou et al. (2001) verificaram que o extrato de quebracho (Schinopsis balansae) rico em taninos diminuiu a viabilidade de larvas de $H$. contortus. Em estudo realizado por Juhnke et al. (2012), foi demonstrado que o uso de extrato de taninos para combater $H$. contortus não só diminuiu o grau de parasitismo como também reduziu o grau de anemia dos animais infectados. Cordeiros quando parasitados e expostos a pastos com diferentes tipos de forrageiras, tendem a consumir plantas com maior teor de tanino (Lisonbee et al., 2009). Essa ingestão ocorre pela associação estabelecida de que o consumo de plantas com elevado teor deste composto reduz a carga parasitária (Lisonbee et al., 2009). Os flavonoides têm ação indireta frente aos parasitas, devido ao fato deste composto ser o maior responsável pela atividade antioxidante da planta; assim sendo, possui efeito direto sobre a redução do envelhecimento celular e melhora da imunidade (Lakshimi et al., 2010).

Avaliar toxicidade dos extratos para os animais e/ou para o meio ambiente é outro ponto-chave que pode e deve ser abordado na metodologia in vitro (Carvalho et al., 2009). Para estabelecer a toxicidade dos fitoterápicos, o ensaio de letalidade frente ao microcrustáceo Artemia salina Leach apresenta-se como um método de alta especificidade (Lhullier et al., 2006). Além de ter menor custo barato, é vendido em forma de cistos, possui distribuição cosmopolita, sendo seguro e prático (Koutsaftis e Aoyama, 2007). Os resultados encontrados nos testes in vitro aliados aos vistos neste teste mostram que o extrato produzido possui propriedades antiparasitárias e ao mesmo tempo não é tóxico ao meio ambiente. Somente após a comprovação de que o herbal não possui toxicidade in vitro o mesmo pode ser aprovado para ser testado in vivo (Arcanjo et al., 2012).

$\mathrm{O}$ extrato demonstrou atividade de inibição da eclodibilidade de ovos com característica dose-dependente e atingiu eficácia superior a $90 \%$ nas concentrações de 50 e $100 \mathrm{mg} / \mathrm{mL}$ (Tabela 1). Diversos compostos fitoterápicos, produzidos a partir de diferentes plantas, já foram testados no TEO com ovos de $H$. contortus. Extratos hidroalcoólicos de Maesa lanceolata e Plectranthus punctatusinibiram $100 \%$ a eclosão de ovos de $H$. contortus quando testados em concentração abaixo de $1 \mathrm{mg} /$ $\mathrm{mL}$ (Tadesse et al., 2009). A mesma concentração inibitória foi observada por Egaule et al. (2011), que testaram extratos aquosos de Leonotis ocymifolia, Leucas martinicensis, Albizia schimperiana e Senna occidentalis. O extrato aquoso de Anacardia humile demonstrou inibição da eclosão dos ovos de $90,9 \%$ na dosagem de $100 \mathrm{mg} / \mathrm{mL}$ (Nery et al., 2010). Extratos aquosos de Syzygium cumini, Genipa americana e Solanumlycocarpum inibiram, respectivamente, 96,17; 18,27 e $14,2 \%$ na concentração de $100 \mathrm{mg} / \mathrm{mL}$ (Oliveira, 2013). Extrato aquoso de Annona senegalensisna concentração $7,1 \mathrm{mg} / \mathrm{mL}$, reduziu a eclodibilidade dos ovos em $88,5 \%$ (Alawa et al., 2003).
Extrato aquoso de Myrsine africana na concentração de $24 \mathrm{mg} /$ $\mathrm{mL}$ obteve eficácia de $77 \%$ (Gathuma et al., 2004). Extrato hidroalcoólico de Tarenaya spinosa inibiu a eclosão de $81,53 \%$ dos ovos do referido parasito, quando utilizado na dosagem de $150 \mathrm{mg} / \mathrm{mL}$ (Andrade et al., 2014).

Tabela 1: Percentual médio ( \pm desvio padrão) de inibição da eclosão de ovos de nematódeos gastrintestinais no teste TEO e do desenvolvimento larval no teste TDL do extrato etanólico obtido de Aloe vera

\begin{tabular}{ccc}
\hline $\begin{array}{c}\text { Concentração } \\
(\mathrm{mg} / \mathrm{mL})\end{array}$ & TEO & TDL \\
\hline 100,0 & $94,35 \pm 1,13 \% \mathrm{~A}$ & $76,03 \pm 0,45 \% \mathrm{~B}$ \\
50,0 & $90,87 \pm 0,58 \% \mathrm{~A}$ & $70,33 \pm 1,56 \% \mathrm{~B}$ \\
25,0 & $66,14 \pm 1,09 \% \mathrm{~B}$ & $55,67 \pm 1,33 \% \mathrm{C}$ \\
12,5 & $53,93 \pm 0,67 \% \mathrm{C}$ & $39,26 \pm 1,14 \% \mathrm{D}$ \\
6,25 & $31,28 \pm 0,78 \% \mathrm{D}$ & $15,24 \pm 2,26 \% \mathrm{E}$ \\
3,12 & $20,03 \pm 0,80 \% \mathrm{E}$ & $5,78 \pm 0,58 \% \mathrm{~F}$ \\
1,56 & $16,07 \pm 1,34 \% \mathrm{EF}$ & $4,24 \pm 1,83 \% \mathrm{~F}$ \\
0,78 & $10,99 \pm 2,54 \% \mathrm{~F}$ & $3,16 \pm 0,77 \% \mathrm{FG}$ \\
$\mathrm{C}-\mathrm{DMSO}(3 \%)$ & $0,79 \pm 0,13 \% \mathrm{G}$ & $0,79 \pm 0,13 \% \mathrm{G}$ \\
$\mathrm{C}+{ }^{\mathrm{TEO}}$ & $97,13 \pm 0,67 \% \mathrm{~A}$ & - \\
$\mathrm{C}+{ }^{\mathrm{TMLA}}$ & - & $97,85 \pm 1,03 \% \mathrm{~A}$ \\
$\mathrm{CL}$ & $11,49 \mathrm{mg} / \mathrm{mL}$ & $22,36 \mathrm{mg} / \mathrm{mL}$ \\
\hline
\end{tabular}

C - = Água destilada; $C+^{\text {TEO }}=$ albendazol $0,63 \mathrm{mg} / \mathrm{mL} ; \mathrm{C}+{ }^{\mathrm{TMLA}}=$ moxidectina $0,63 \mathrm{mg} / \mathrm{mL} ; \mathrm{CL}_{50}=$ concentração letal para $50 \%$ dos ovos. Letras maiúsculas comparam médias nas colunas; letras dessemelhantes indicam diferença significativa $(P<0,05)$.

O fitoterápico não inibiu mais de $90 \%$ o desenvolvimento larval em nenhuma das concentrações testadas, sendo seu efeito igualmente dose-dependente (Tabela 1). É importante enfatizar que os resultados encontrados no TEO são superiores ao TDL, em todas as concentrações testadas $(p<0,05)$, o que também foi observado na literatura científica consultada. Extratos aquosos de Leonotis ocymifolia, Leucas martinicensis, Albizia schimperiana e Senna occidentalis induziu 100, 99,85, 99,31 e $96,36 \%$ de inibição do desenvolvimento larval de $H$. contortus, respectivamente (Egaule et al., 2011). Em estudo conduzido por Macedo et al. (2009), utilizando óleo essencial de Eucalyptus globulus na concentração de $43,5 \mathrm{mg} / \mathrm{mL}$, foi demonstrado $98,7 \%$ de inibição do desenvolvimento larval. Extrato aquoso de Musa sp., avaliado na concentração de $57,76 \mathrm{mg} / \mathrm{mL}$ apresentou eficácia de 90\% (Oliveira et al., 2010). Extratos de folhas e sementes produzidos com acetona, etilacetato e metanol produzidos com Annona squamosa, Eclipta prostrata, Solanum torvum, Terminalia chebula e Catharanthus roseus mostraram completa inibição (100\%) quando utilizados na concentração de $50 \mathrm{mg} / \mathrm{mL}$ (Kamaraj e Rahuman, 2011). Utilizando a concentração de $50 \mathrm{mg} / \mathrm{mL}$ de extrato acetato de etila produzido com Azadirachta indica observou-se inibição no desenvolvimento larvar de 68,10\% (Costa et al., 2008). 
Mesmo existindo diversos estudos acerca das propriedades biológicas da $A$. vera, nunca houve pesquisas focando na ação da planta frente $H$. contortus. Essa planta possui diversas finalidades já documentadas. Todavia, muitas serão elucidadas devido à grande quantidade de compostos presentes em sua estrutura (Boudreau e Beland, 2006; Cellini et al., 2014). O extrato demonstrou desempenho promissor, mesmo quando comparados com os controles positivos, o que evidencia a potente atividade antiparasitária da planta.

\section{Referências}

ALAWA, C.B.; ADAMU, A.M.; GEFU, J.O.; AJANUSI, O.J.; ABDU, P.A.; CHIEZEY, N.P.; ALAWA, J.N.; BOWMAN, D.D. In vitro screening of two Nigerian medicinal plants (Vernonia amygdalina and Annona senegalensis) for anthelmintic activity. Veterinary Parasitology, v.113, p.73-81, 2003.

ALONSO-DÍAZ, M.A.; TORRES-ACOSTA, J.F.J.; SANDOVALCASTRO, C.A.; HOSTE, H. Comparing the sensitivity of two in vitro assays to evaluate the anthelmintic activity of tropical tannin rich plant extracts against Haemonchus contortus. Veterinary Parasitology, v.181, p. 360-364, 2011.

ANDRADE, F.D.; RIBEIRO, A.R.C.; MEDEIROS, M.C.; FONSECA, S.S.; ATHAYDE, A.C.R.; FERREIRA, A.F.; ONALDO, G.R.; SILVA, W.W. Anthelmintic action of the hydroalcoholic extract of the root of Tarenaya spinosa (Jacq.) Raf. for Haemonchus contortus control in sheep. Pesquisa Veterinária Brasileira, v. 34, p. 942-946, 2014.

ARCANJO, D.D.R.; ALBUQUERQUE, A.C.M.; MELO-NETO, B.; SANTANA, L.C.L.R.; MEDEIROS, M.; CITÓ, A.M.G.L. Bioactivity evaluation against Artemia salina Leach of medicinal plants used in Brazilian Northeastern folk medicine. Brazilian Journal of Biology, v.72, n. 3, p. 505-509, 2012.

ATHANASIADOU, S.; KYRIAZAKIS, I.; JACKSON, F.; COOP, R.L. Direct anthelmintic effects of condensed tannins towards different gastrointestinal nematodes of sheep:in vitro and in vivo studies. Veterinary Parasitology, v. 99, p. 205-219, 2001.

BIZIMENYERA, E.S.; GITHIORI, J.B.; ELOFF, J.N.; SWAN, G.E. In vitro activity of Peltophorum africanum Sond. (Fabacea) extracts on the egg hatching and larval development of the parasitic nematode Trichostrongylus colubriformis. Veterinary Parasitolology, v.142, p. 336-343, 2006.

BLOIS, M.S. Antioxidant determinations by the use of a stable free radical. Nature, v.181, p.1199-1200, 1958.

BOUDREAU, M.D.; BELAND, F. A. An evaluation of the biological and toxicological properties of Aloe barbadensis (miller), Aloe vera. Journal of Environmental Science and Health Part C, v.24, n.1, p.103-154, 2006.

CELLINI, L; DI BARTOLOMEO, S.; DI CAMPLI, E.; GENOVESE, S.; LOCATELLI, M.; DI GIULIO, M. In vitro activity of Aloe vera inner gel against Helicobacter pylori strains. Letters in applied microbiology, v.59, n.1, p. 43-48, 2014.

COLES, G.C.; BAUER, C.; BORGSTEEDE, F.H.M.; GEERTS, S.; KLEI, T.R.; TAYLOR, M.A.; WALLER, P.J. World Association for the Advancement of Veterinary Parasitology (WAAVP) - methods for detection of anthelmintic resistance in nematodes of veterinary importance. Veterinary Parasitolology, v. 44, p. 35-44, 1992.

\section{Conclusão}

Concluiu-se que o extrato obtido pela metodologia descrita no presente experimento demonstrou ser candidato a fitoterápico antiparasitário frente $H$. contortus. Os exames bioquímicos apontaram a presença de diversos compostos químicos que possuem a capacidade nematocida. Novos estudos devem ser realizados buscando maximizar a eficácia do produto e posteriormente testá-lo em testes in vivo.

COSTA, C.T.C.; BEVILAQUA, C.M.L.; CAMURÇA-

VASCONCELOS, A.L.F.; MACIEL, M.V.; MORAIS, S.M.;

CASTRO, C.M.S.; BRAGA, R.R.; OLIVEIRA, L.M.B. In vitro ovicidal and larvicidal activity of Azadirachta indica extracts on Haemonchus contortus. Small Ruminant Research, v. 74, p. 284287, 2008.

DAGNE, E.; BISRAT, D.; VILJOEN, A.; VAN WYK, B.E. Chemistry of Aloe species. Current Organic Chemistry, v. 4, n.10, p.10551078, 2000.

FORTES, F.S.; KLOSTER, F.S.; SCHAFER, A.S.; BIER, D.; BUZATTI, A.; YOSHITANI, U.Y.; MOLENTO, M.B. Avaliação da resistência em um isolado de campo selecionado de Haemonchus contortus à ivermectina e moxidectina usando o Teste de Migração de Larvas em Ágar. Pesquisa Veterinária. Brasileira, v. 33, p.183-187, 2014.

GATHUMA, J.M.; MBARIA, J.M.; WANYAMA, J.; KABURIA, H.F.A.; MPOKE, L.; MWANGI, J.N. Efficacy of Myrsine africana, Albizia anthelmintica and Hilderbrantia sepalosa herbal remedies against mixed natural sheep helminthosis in Samburu district, Kenya. Journal of Ethnopharmacology, v. 91, p. 7-12, 2004.

HOSTE, H.; JACKSON, F.; ATHANASIADOU, S.; THAMSBORG, S.M.; HOSKIN, S.O. The effects of tannin-rich plants on parasitic nematodes in ruminants. Trends in Parasitology, v.22, p. 253-261, 2006.

Hubert, J.; Kerboeuf, D. A microlarval development assay for the detection of anthelmintic resistance in sheep nematodes. Veterinary Record, v.130, p. 442-446, 1992.

HUSEINI, H.F.; KIANBAKHT, S.; HAJIAGHAEE, R.; DABAGHIAN, F. H. Anti-hyperglycemic and anti-hypercholesterolemic effects of Aloe vera leaf gel in hyperlipidemic type 2 diabetic patients: a randomized double-blind placebo-controlled clinical trial. Planta Medica-Natural Products and MedicinalPlant Research, v.78, n. 4, p. 311, 2012.

JUHNKE, J.; MILLER, J.; HALL, J.O.; PROVENZA, F.D.; VILLALBA, J. J. Preference for condensed tannins by sheep in response to challenge infection with Haemonchus contortus. Veterinary parasitology, v.188, n.1, p.104-114, 2012.

KAMARAJ, C.; RAHUMAN, A.A. Efficacy of anthelmintic properties of medicinal plant extracts against Haemonchus contortus. Research in veterinary science, v. 91, n. 3, p.400-404, 2011.

KAYSER, O.; KIDERLEN, A.F.; CROFT, S. L. Natural products as antiparasitic drugs. Parasitology Research, v.90, n. 2, p. 55-62, 2003.

KOUTSAFTIS, A.; AOYAMA, I. Toxicity of four antifouling biocides and their mixtures on the brine shrimp Artemia salina. Science of the Total Environment, v. 387, n.1, p.166-174, 2007. 
LHULLIER, C.; HORTA, P.A.; FALKENBERG, M. Avaliação de extratos de macroalgas bênticas do litoral catarinense utilizando o teste de letalidade para Artemia salina. Revista Brasileira de Farmacognosia, v.16, p.158-163, 2006.

LISONBEE, L.D.; VILLALBA, J.J.; PROVENZA, F.D.; HALL, J.O. Tannins and self-medication: Implications for sustainable parasite control in herbivores. Behavior Processes, v. 82, p.184189, 2009.

MACEDO, I.T.; BEVILAQUA, C.M.; OLIVEIRA, L.D.; CAMURÇAVASCONCELOS, A.L.; VIEIRA, L.D.S.; OLIVEIRA, F.R.; BARROS, R.S.; CHAGAS, A.C. Atividade ovicida e larvicida in vitro do óleo essencial de Eucalyptus globulus sobre Haemonchus contortus. Revista Brasileira de Parasitologia Veterinária, v.18, p. 62-66, 2009.

MATOS, F.J.A. Farmácias vivas. 3. ed. Fortaleza: Edições UFC, 1998. 220 p.

MCDONALD, S.; PRENZLER, P.D.; ANTOLOVICH, M.; ROBARDS, K. Phenolic content and antioxidant activity of olive extracts. Food Chemistry, v.73, p.73-84, 2001.

MEYER, B.N.; FERRIGNI, N.R.; PUTNAM, J.E.; JACOBSEN, L.B.; NICHOLS, D.E.; MCLAUGHLIN, J.L. Brine shrimp: a convenient general bioassay for active plant constituents. Plant Medica, v. 45, p. 31-34, 1982.

MOLAN, A.L.; DUNCAN, A.J.; BARRY, T.N.; MCNABB, W.C. Effects of condensed tannins and crude sesquiterpene lactones extracted from chicory on the motility of larvae of deer lungworm and gastrointestinal nematodes. Parasitology, v. 52, p.209-218, 2003.

MOLENTO, M.B. Resistência parasitaria em helmintos de equídeos e propostas de manejo. Ciência Rural, v. 35, p.14691477, 2005.

MOLENTO, M.B.; VERÍSSIMO, C.J.; AMARANTE, A.T.; VAN WYK, J.; CHAGAS, A.C.S.; DE ARAÚJO, J.V.; BORGES, F.A. Alternativas para o controle de nematoides gastrintestinais de pequenos ruminantes. Arquivos do Instituto Biológico, v. 80, n. 2, p. 253-263, 2013.

NERY, P.S.; NOGUEIRA, F.A.; MARTINS, E.R.; DUARTE, E.R. Effects of Anacardium humile leaf extracts on the development of gastrointestinal nematode larvae of sheep. Veterinary Parasitology, v.171, p. 361-364, 2010.
OLIVEIRA, L.D.R. Plantas medicinais como alternativa para o controle de Haemonchus contortus em ovinos: testes in vitro e in vivo, 2013. 59 p. Dissertação (Mestrado) - Ciências Animais Universidade de Brasília, Brasília, 2013.

OLIVEIRA, L.N.; DUARTE, E.R.; NOGUEIRA, F.A.; SILVA, R.B.; FILHO, D.E.F; GERASEEV, L.C. Eficácia de resíduos da bananicultura sobre a inibição do desenvolvimento larval em Haemonchus spp. provenientes de ovinos. Ciência Rural, v. 40, p. 488-490, 2010.

RAJASEKARAN, S.; RAVI, K.; SIVAGNANAM, K.; SUBRAMANIAN, S. Beneficial effects of Aloe vera leaf gel extract on lipid profile status in rats with streptozotocin diabetes. Clinical and Experimental Pharmacology and Physiology, v. 33, n. 3, p. 232-237, 2006.

RAMOS, A.P.; PIMENTEL, L.C. Ação da Babosa no reparo tecidual e cicatrização. Brazilian Journal of Health, v. 2, n.1, 2013

ROBERTS, F.H.S; O'SULLIVAN, P.J. Methods for egg counts and larval cultures for strongyles infesting the gastro-intestinal tract of cattle. Crop Pasture Science, v.1, p. 99-102, 1950.

SPRENGER, L.K.; AMARAL, C.H.; LEITE FILHO, R.V.; AGUIAR, T.N.; MOLENTO, M. B. Eficácia do fosfato de levamisol em nematódeos gastrintestinais de caprinos e ovinos. Archives of Veterinary Science, v.18, n.1, p. 29-39, 2013.

SPRENGER, L.K.; BUZATTI, A.; CAMPESTRINI, L.H; YAMASSAKI, F.T.; MAURER, J.B.B.; BAGGIO, S.F.Z.; MAGALHÃES, P.M.; MOLENTO, M.B. Atividade ovicida e larvicida do extrato hidroalcoólico de Artemisia annua sobre parasitas gastrintestinais de bovinos. Arquivo Brasileiro de Medicina Veterinária e Zootecnia, v. 67, n.1, p. 25-31, 2015.

TADESSE, D.; EGUALE, T.; GIDAY, M.; MUSSA, A. Ovicidal and larvicidal activity of crude extracts of Maesa lanceolata and Plectranthus punctatus against Haemonchus contortus. Journal of ethnopharmacology, v.122, n. 2, p. 240-244, 2009.

YIM, D.; KANG, S.S.; KIM, D.W.; KIM, S.H.; LILLEHOJ, H.S.; MIN, W. Protective effects of Aloe vera-based diets in Eimeria maxima-infected broiler chickens. Experimental Parasitology, v.127, n.1, p.322-325, 2011. 